\title{
CRÍTICA A LOS MÓDULOS “AMOR Y SEXUALIDAD” DE LA CONFERENCIA EPISCOPAL PARA EDUCAR SOBRE SEXUALIDAD
}

\section{REFLECTION ABOUT THE DOCUMENTS "LOVE AND SEXUALITY” OF THE EPISCOPAL CONFERENCE TO EDUCATE ABOUT SEXUALITY}

\author{
Paula Sequeira Rovira*
}

RESUMEN

Este artículo plantea una problematización a los seis módulos llamados "Amor y Sexualidad", que sacó a la luz, la iglesia católica costarricense, en el 2009, para enseñar sobre el tema de sexualidad. Los textos son un interesante material para analizar dado la influencia de la jerarquía católica en muchas de las decisiones concernientes a temas relacionados con la sexualidad en Costa Rica. No solo se analizan los contenidos teóricos presentes en los módulos, sino que se detallan algunas contradicciones encontradas.

PALABRAS CLAVE: SEXUALIDAD * CATOLICISMO * ANTICONCEPCIÓN * HOMOSEXUALIDAD * XENOFOBIA

\section{ABSTRACT}

This article raises the six documents called "Love and Sexuality", which brought to light, the Catholic Church of Costa Rica, in 2009, to teach on the subject of sexuality. The texts are an interesting material for analysis, given the influence of the Catholic hierarchy in many decisions concerning issues related to sexuality in Costa Rica. The article not only analyzes the theoretical content present in the modules, also points out contradictions found in the documents.

KEYWORDS: SEXUALITY $*$ CATHOLICISM $*$ CONTRACEPTION $*$ HOMOSEXUALITY $*$ XENOPHOBIA

Instituto de Estudios de la Mujer y Escuela Ecuménicas de Ciencias de la Religión de la Universidad Nacional (Costa Rica).

paulasr30@yahoo.com 


\section{INTRODUCCIÓN}

En el 2009, la Conferencia Episcopal de Costa Rica (CECOR), sacó a la luz una serie de seis fascículos titulados "Amor y Sexualidad", cada uno de los cuales proponía rangos de edad para trabajar dichos temas, que iban desde los 0 hasta los 18 y más años. Según informaciones dadas a conocer en la prensa escrita, dichos documentos surgieron porque se produjo una "ruptura de conversaciones con el Ministerio de Educación Pública sobre ese tema, [que] motivó a los obispos a acelerar la producción de sus propias guías" (Ávalos, 2009a). No es ningún secreto que la influencia de la jerarquía católica costarricense en la educación en sexualidad impartida en colegios y escuelas tiene larga data $^{1}$. El peso de esta entidad en la institucionalidad de nuestro país sigue siendo poderoso.

El público meta de los módulos, son en primer lugar, los y las estudiantes de escuelas y colegios católicos de 70 centros educativos a lo largo del país (Ávalos, 2009b). Además, según se informó, cinco mil de estos documentos se repartirán a diócesis para que sean utilizados por pastorales familiares y juveniles (ídem). Los textos fueron elaborados por educadores(as) y revisados por dos sacerdotes, un diácono, una educadora, una curriculista y Monseñor Vittorino Girardi, obispo de Tilarán-Liberia.

El siguiente trabajo tiene como propósito analizar las visiones sobre la sexualidad presentes en los seis módulos, elaborados por la Conferencia Episcopal en el 2009, esto con el fin de producir debates académicos al respecto, que promuevan la crítica constructiva de estas posiciones. Por lo tanto, el trabajo se dividirá de la siguiente manera: en una primera parte, donde se analizan los conceptos fundamentales de estos documentos y una crítica a los mismos; en segundo lugar, se señalan algunas contradicciones encontradas a lo largo de la lectura realizada $y$ finalmente, se hacen conclusiones de lo desarrollado.

$1 \quad \mathrm{Al}$ respecto, ver el aporte que hace Isabel Gamboa (2009), en su libro El sexo como lo cura, en la recuperación de esta historia.

\section{DECONSTRUYENDO LOS TEXTOS: ALGUNAS REFLEXIONES AL RESPECTO}

De acuerdo con el sociólogo e historiador inglés, Jeffrey Weeks (1998), la forma en que pensamos acerca del sexo tiene un impacto directo en cómo lo vivimos. Por ello es importante entender que para los textos aquí analizados, la sexualidad se presenta como un "misterio sagrado" (CECOR, 2009: 8, v. 1), aunque más adelante también será definida como:

...un elemento básico de la personalidad; un modo propio de ser, de manifestarse, de comunicarse con los otros, de sentir, expresarse y vivir el amor humano. Esta capacidad de amar como don de sí tiene, por tanto, su "encarnación" en el carácter esponsal del cuerpo, en el cual está inscrita la masculinidad y la feminidad de la persona (SH 10) (CECOR, 2009: 17, v. 1).

En los textos, la sexualidad está inapelablemente ligada al amor: una sin otra, no son bien vistas y como se dice, es una característica "irrenunciable" de la misma (CECOR, 2009: 17, v. 1). Desde el título que da vida a los módulos hasta los contenidos presentados en ellos, "el amor" tiene un carácter protagónico que de alguna manera, busca transversalizar los temas tratados.

Aunque en los documentos se haga una correlación tan automática de amor y sexualidad, la primera, solo de manera muy reciente, ha estado vinculada a la segunda, por lo menos en la historia occidental. De acuerdo con el sociólogo Anthony Giddens (2000: 44-45), en Europa premoderna, muchos de los casamientos se realizaban por contrato para solventar problemas económicos y como él mismo señala, en el siglo XVII, los besos y caricias eran "raras" entre parejas casadas. Por esto, al hacer una asociación tan inmediata, se esconde que este ha sido un fenómeno creado de manera más o menos reciente $y$ que a su vez, tiene matices diferentes para las culturas y también para los géneros ${ }^{2}$.

2 Ver Jiménez Guzmán y Tena Guerrero (2001), por los interesantes argumentos sobre el amor, el género y la negociación coital. 
Si como dicen los textos, “... la sexualidad apunta a la realización conyugal en el matrimonio" (CECOR, 2009: 18, v. 1), esto querría decir que la misma es vista como un acto muy específico, posiblemente relacionado con el coito y no como una parte constitutiva de la vida humana. Desde una perspectiva holística, la realización sexual no tendría porque estar en el matrimonio porque la sexualidad no se limita a una sola práctica, ni mucho menos a un solo rito. Esta aseveración también desconoce la diversificación de expresiones sexuales, los nuevos arreglos de parejas existentes y a su vez, promueve una visión reduccionista del término. En todos los documentos, la sexualidad es vista de manera ahistórica y homogénea. No parecen derivarse del planteamiento, diferencias en su vivencia relacionadas con ninguna característica humana.

Claramente se demuestra cómo los documentos tratan a todas las personas como unidades homogéneas y armónicas. Según es señalado: "Sólo existen dos formas de ser 'ser humano': hombre o mujer" (CECOR, 2009: 73, v. 1). Así, aunque la feminidad y masculinidad son reseñadas como "naturales" e innatas, también se dice en otra parte el documento:

Desde antes de nacer el niño o la niña vienen dotados de condiciones físicas que les distinguen como hombre o como mujer. Sin embargo, a partir del nacimiento, tal condición debe ser educada, construida e interiorizada por él o ella, hasta llegar a tener conciencia clara de su identidad sexual (CECOR, 2009: 49-50, v. 1).

Pero, si se estuviera seguro(a) de que la feminidad, masculinidad y la identidad sexual son "naturales" y esencias del ser, ¿por qué habría que educar estas características?, ¿para qué promover su construcción y mantenimiento por parte de los/as progenitores? A pesar de que el texto señala que "Ser hombre, ser mujer es una realidad buena y querida por Dios" (CECOR, 2009: 14, v. 1), el mismo no especifica cuáles son estas características que "definen" a unos y a otras. Esto se debe probablemente a que se crea que por ser "naturales" todas las personas las saben de antemano ${ }^{3}$ El hecho que se diga que tanto la masculinidad como la feminidad son "complementarias", genera que no se deje espacio para otras posibilidades de expresiones de amor o deseo entre las personas que no se identifican como heterosexuales.

Todos los módulos están plagados de dicotomías jerarquizadas: mujer/hombre, masculinidad/feminidad, sexo bueno/sexo malo. Se promulgan solo dos formas de vivir la sexualidad: una correcta y "querida por Dios" y otra incorrecta y cargada de "pecado". Como es recordado por Vendrell Ferré (2004: 90), el modelo implantado de sexualidad necesita la creación de estas dicotomías para controlar los actos sexuales permitidos. Una sexualidad "normal", necesita de una "anormal", para funcionar correctamente.

También merece la pena recordar que entre las complicaciones más grandes de los textos, están no solo, las que invisibilizan completamente los procesos históricos que han formado parte en la constitución de la sexualidad y su importancia en Occidente, sino que a su vez, también el documento es ciego a mirar la realidad de Costa Rica y en el mundo, en cuanto a la sexualidad y los cambios que se han desarrollado en estos aspectos en las sociedades. Por ejemplo, para mencionar solo algunos datos, se sabe que el inicio de las "relaciones sexuales" en la región se da en promedio a los 14 años y además el $50 \%$ de los(as) jóvenes menores de 17 años se declaran sexualmente activos(as) (Organización Panamericana de la Salud, 2002: 200), asimismo, en Costa Rica la prevalencia anticonceptiva es del $80 \%$ y el porcentaje de embarazos no deseados es del 42\% (Chen, et ál.: 2001). A la visión armónica de los textos no le interesa hacer una mirada al entorno y esto sesga la vivencia de millones de personas que escogen vivir otros tipos de vida sexual, diferentes a los propuestos en ellos.

3 De acuerdo con Butler (2007), la categoría mujeres ( $y$ por lo tanto también la de hombres) ha pasado a ser un término problemático. Dar por sentado las características de unas o de otros es caer en posiciones esencialistas $y$ universalistas $y$ produce reificaciones de las relaciones sociales entre las personas. 
Aunque pareciera que los textos aquí analizados hablan poco del deseo, lo cierto es que este se encuentra por todos lados. El deseo es visto como peligroso y subversivo, este se comienza a "corregir" desde los 6 meses cuando los(as) infantes comienzan a auto erotizarse. Los(as) progenitores deben estar avocados a la tarea de promover la "subordinación de las dimensiones instintivas", la sexualidad debe ser vivida en "castidad", "virginidad" o en el matrimonio; el pudor es exaltado como una virtud porque promueve la "negativa a mostrar lo que debe permanecer oculto". Se manifiesta luego de leer el texto, un miedo latente al deseo, creyendo que de alguna manera este se desbordará (estos argumentos fueron lo que en su momento, Gayle Rubin (1989: 141) llamó "la teoría del dominó del peligro sexual”); para los documentos, la sexualidad y sus expresiones, en la cultura occidental, siempre deben de estar cuidadas, vigiladas y controladas (Foucault, 1989). En un primer momento, pareciera que esta tarea le corresponde a los padres y madres y que luego al crecer, le tocará a cada quien recordar "las consecuencias del pecado original". Este miedo, promueve una visión negativa del deseo y de las expresiones de la sexualidad, que no ayuda a promover una mirada humana de la misma $y$ mucho menos a formarla de manera integral.

Parece fundamental recordar las cinco "recomendaciones" presentes en los documentos, para trabajar el tema de la sexualidad, las cuales son: (1) Tener presente "las consecuencias del pecado original, es decir, la debilidad humana y la necesidad de gracia de Dios para superar las tentaciones y evitar el pecado"; (2) Recordar que la información suministrada debe ser adecuada para el desarrollo individual de la población meta, en este caso, niños, niñas y jóvenes; (3) Hablar de tres problemas específicos los cuales son: a) el orden en que se debe conversar sobre sexualidad (fertilidad, regulación natural de dicha fertilidad basada en el amor, fidelidad, procreación de acuerdo al plan divino y por último, respeto a la vida humana), b) no abordar el tema de la homosexualidad a menos que "surja algún específico problema grave" y c) se aconseja que "las desviaciones sexuales ${ }^{4 "}$ se traten solo a través de consejos individuales; al hablar se recuerda saber elegir el momento correcto para tratar los problemas específicos; (4) No mostrar ningún documento "de naturaleza erótica [erotizante]" a la población aquí tratada; por último, (5) Exhortar a que no se debe obligar a nadie a actuar de forma que pueda "lesionar subjetivamente su propia delicadeza y el sentido de su intimidad" (CECOR, 2009:12-13, v. 1).

Todo lo anteriormente esbozado, hace entrever que los documentos tienen un claro "enfoque esencialista 5 " pues, como dice Weeks, este tipo de orientación:

... intenta explicar las propiedades de una totalidad compleja basándose en una supuesta verdad interna o una esencia, en la suposición de que "en todos los asuntos sexológicos debe haber un esquema único, básico, uniforme decretado por la naturaleza misma" (Weeks, 1998).

4 Para los textos, la identidad sexual "buena" es la heterosexual, por lo tanto señala: "Así pues, a eso de los tres años, tanto las niñas como los niños deben haber elaborado lo fundamental de su identidad sexual... Si a esa edad no hubiera logrado la construcción básica de su identidad sexual, entonces se hace necesario algún tipo terapéutico para apoyarle en la elaboración de su núcleo de identidad sexual" (CECOR, 2009: 50, v. 1). La homosexualidad $y$ lesbianismo, son vistos como negativos y no reciben aprobación, pues se contraponen a "la ley natural" al cerrar "el acto sexual al don de la vida” (CECOR, 2009: 32, v. 4).

5 Aunque, de acuerdo con todos los documentos, el enfoque utilizado es llamado "personalizante", explicado como aquel que "parte de la concepción integral del ser humano $y$ tiene en cuenta diversos elementos esenciales de la persona, como son la libertad, la autonomía, la responsabilidad, la sociabilidad, la capacidad de amar, la voluntad, entre otros" (CECOR, 2009: 7, v. 1). Más adelante se hablará que la sexualidad también es pensada desde un enfoque "ecológico-contextual", el cual se dice que está basado en planteamientos de Vigotsky, donde se toma en cuenta "los procesos de socialización en el desarrollo de las personas" (CECOR, 2009: 8, v. 1), pero estos no se desarrollan con más detenimiento. 
Los textos mencionados no solo dejan ideas vagas e imprecisas, por la poca profundización de conceptos que sustenta el pensamiento analizado, sino que además es interesante notar que existe un aura de secretismo en el abordaje de la sexualidad. Se recomienda, en varias ocasiones, no hablar de la homosexualidad, tratar el tema de las desviaciones sexuales solo de forma individual, no mostrar imágenes “eróticas”. En el fondo, ¿qué está diciendo esta idea a ocultar? ¿Por qué el afán de dejar ciertos asuntos solo si "surge algún problema grave"?

Se concluye en esta primera parte, que una educación en sexualidad que promueva relaciones más equitativas entre las personas no debería tener este tipo de visiones y el desconocimiento de muchas otras realidades que claramente se omiten en los documentos. La sexualidad tratada como "misterio", como un secreto, solo promueve el desconocimiento y la falta de información clara para las personas $y$ las consecuencias de esto, las seguimos viendo todos los días.

\section{CONTRADICCIONES E INCOHERENCIAS ENCONTRADAS}

Cuando se leen con detenimiento los módulos aquí planteados, se hacen evidentes algunas contradicciones, no menores, las cuales se consideran necesarias de reseñar. A continuación se señalarán solo dos de ellas:

1) Uno de los aspectos pilares de todo el documento es aquel que señala que solo existen hombres y mujeres y recíprocamente, masculinidad y feminidad. Este argumento por simple que parezca, representa una parte fundamental de las argumentaciones propuestas en los módulos. Pero, lo interesante es cuando se analiza esta idea con otra propuesta en el Módulo 2:

Ahora bien, en caso todos los casos, "los hombres nacen con genitales masculinos $y$ los cromosomas XY, mientras que las mujeres poseen genitales femeninos y los dos cromosomas X. Sin embargo, existen personas que no pueden ser clasificadas por estos factores, ya que poseen combinaciones de cromosomas, hormonas $y$ genitales que no siguen las definiciones típicas que se han relacionado con el varón y la mujer (Identidad sexual, es.wikipedia.com) (CECOR, 2009: 50, v. 2).

Esta pequeña referencia tomada de Wikipedia, cambia todo el sentido y la argumentación de todos los textos. Los módulos basan buena parte de su razonamiento, en la idea de que solo existen dos sexos (complementarios): "hombres" y "mujeres", esto hace que desde este enfoque, los primeros tengan una "masculinidad" y las segundas una "feminidad" (CECOR, 2009: 73, v. 1). Estas atribuciones son miradas en el texto sobre todo de forma esencialista y dicotómicas, pues como se señala: “... al jugar con una muñeca la niña lo hace como una mujer y el niño lo hace como varón" (CECOR, 2009: 49, v. 2). Pero si existe la posibilidad, como se plantea en el Módulo 2, de personas que presenten características que no muestren lo que típicamente se piensa como hombre o mujer, la argumentación se vendría abajo. La incursión en la escena de las personas intersexuales ${ }^{6}$ haría tambalear los cimientos argumentativos de todas las "guías". De esta manera, no importa, si existe una o un millón de personas intersex ${ }^{7}$, pues con solo que existan "algunas" personas que trascienden el esquema tradicional de los hombres y las mujeres, muchas de las otras argumentaciones dejan de tener sentido o por lo menos pierden fuerza, complementariedad, naturaleza de la feminidad/ masculinidad, la posibilidad de solo dos opciones, etc.

2) Otra contradicción interesante $y$ de fuertes implicaciones tiene que ver con el postulado de la identidad sexual. En todos los documentos, es claro mirar como la heterosexualidad es ensalzada y promovida como

$6 \quad$ Las personas intersexuales o intersex son quienes tiene un "sexo ambiguo" (Maffia y Cabral, 2003) y que en el sistema médico suelen sufrir de "disciplinamiento quirúrgico" porque el pensamiento dicotómico hace creer que solo existen los hombres o las mujeres.

7 De acuerdo con algunos datos, la intersexualidad se produciría en 1 de cada 2000 nacimientos (Wikipedia). 
"querida por Dios" y como natural. Aun así, en varios extractos de los documentos se muestra incoherencias interesantes. Haciendo referencia nuevamente a Wikipedia, se señala "La identidad sexual es determinada por 'multitud de factores, entre los que podemos destacar el psicológico, social ${ }^{8}$ y biológico"' (CECOR, 2009: 49, v. 2). La contradicción se hace evidente al leer la cita anterior, pues si se supone que la identidad sexual tiene que ver con elementos sociales entonces no podría hablarse de una naturalidad de la heterosexualidad. Como se señala en otra parte de los documentos, a partir del nacimiento, la condición de ser "hombre" o "mujer" "debe ser educada, construida e interiorizada por él o ella, hasta llegar a tener conciencia clara de su identidad sexual" (CECOR, 2009: 49-50, v. 1) ¿Por qué tendría que educarse algo que es innato?

Saltan a la luz las preguntas, luego de mostrar el panorama anterior, de ¿por qué dejar tantos cabos sueltos?, ¿cuáles serían las razones que llevan a quienes escribieron estos documentos a hacer argumentaciones fácilmente refutables? Algunas hipótesis que se plantean en este trabajo, por las que esto podría estar ocurriendo, serían las siguientes:

a) Una primera explicación podría deberse a la falta de tiempo para realizar los documentos, lo que les imposibilitó corroborar que no quedaran "cabos sueltos" teóricos. Aunque esto pudiera o no tener algo de verdad, no es una explicación que aclare el trasfondo de estas incoherencias o contradicciones.

b) Otra explicación podría deberse a que quienes hicieron el material, tienen poco conocimiento sobre la sexualidad $y$ que sus razonamientos los hacen basados en la "creencia religiosa" y no en la científica. El texto está plagado de poca

$8 \quad$ La cursiva es propia.

9 Esta misma confusión se daría con otro término relacionado que es el de "orientación sexual" que, aunque en uno de los módulos el mismo es visto como una "invención" (CECOR, 2009: 50, v. 2), en otro, se concuerda con el término (CECOR, 2009: 25, v. 6). claridad en las explicaciones, muchas de las cuales se basan en textos elaborados por sacerdotes, obispos u otros creyentes católicos(as), pero que no necesariamente tienen conocimientos en sexualidad. Por ejemplo, esto se puede notar claramente, cuando hablan de abuso sexual o de la orientación sexual. Con respecto al abuso sexual, aunque es un tema importante, es realmente poco abordado y llama la atención que cuando se le trata, se hacen apreciaciones como que uno de los síntomas o indicadores que muestran que se está dando alguna clase de agresión sexual contra un niño o niña es la "ausencia de formación religiosa" (CECOR, 2009: 69, v. 2).

c) Otra posible hipótesis que explique estas contradicciones tendría que ver con que se utilizaron argumentaciones basadas en Freud o en la Wikipedia, las cuales posiblemente se emplearon para no ser posteriormente tildados de tradicionales. Una de las fuertes críticas que se le señala a la jerarquía de la iglesia católica es que suelen ser "conservadores" o "anticuados" en sus perspectivas con respecto a la sexualidad $y$ esto hace que algunos fieles se alejen (Arangurem, 1996: 35; Jiménez y Osuna, 2007: 6). La contraposición de los pensamientos religiosos con otros textos como Wikipedia co ayudan a que el texto sea poco claro y contradictorio.

d) Por último, es posible que hayan utilizado la estrategia de confundir con la misma confusión. Existe en el texto toda una serie de ideas poco claras y no explicadas como "pasiones desordenadas", "pudor", "lo querido por Dios", "encauzar correctamente los sentimientos o pasiones", "edad de la inocencia", "promiscuidad", "decisiones adecuadas", "conductas sexuales inapropiadas", "orden moral objetivo" (entre muchas otras), que lleva a la confusión y a la poca precisión. Más que posturas argumentativas claras, designan posiciones subjetivas y desde el sentido común. No aclaran, sino más 
bien proveen un "limbo argumentativo" que deja el portillo abierto para incluir en ellas cualquier interpretación posible.

\section{IV. ¿QUÉ TIENE QUE VER LA XENOFOBIA Y LA SEXUALIDAD?}

Una parte de los documentos aquí analizados merece especial atención por su relación con comentarios claramente xenofóbicos. Pero ¿qué tiene que ver la xenofobia con la sexualidad?

En los textos, uno de los postulados más férreamente defendidos tiene que ver con la negativa de utilizar métodos anticonceptivos no naturales. De acuerdo con el documento, la anticoncepción responde a una "mentalidad hedonista" (CECOR, 2009: 75, v. 4) y se oponen a ella porque quienes la utilizan "se atribuyen el derecho indiscriminado de ser árbitros de la vida" (ídem). El problema principal de los anticonceptivos resulta ser el "negar la vida". Específicamente en el módulo número 5, existen a partir de la página 77 y subsiguientes, interesantes comentarios tomados del mexicano Luis García Pimentel, quien estaba preocupado por el tema de la anticoncepción y la "sobrepoblación" desde un enfoque católico. Él escribió un libro publicado La falacia del Simio, que nutre mucho de la argumentación con la que se defiende esta posición contra la anticoncepción. Sus razonamientos se basan en poner en duda el hecho que el control de la natalidad tenga efectos positivos en el mundo y que solucione el problema de sobrepoblación, porque según su reflexión, existe mucha riqueza en el mundo para alimentar a las personas que viven en la pobreza o en la miseria. Esto es lo que él llamó "la falacia del simio" en la cual la población mundial podría subsistir con los recursos actuales $y$ en sus palabras "... el control artificial de la natalidad, en lugar de combatir la sobrepoblación y apuntalar el mantenimiento sano del género humano, enferma a la sociedad, estimula el desequilibrio entre producción y población y deshace la riqueza de las poblaciones" (García, citado en CECOR, 2009: 78, v. 5).

$\mathrm{Su}$ argumentación seguirá en una metáfora animalesca entre quienes se expanden $y$ quienes se extinguen, entre quienes son "aptos" y quienes no. Desde su punto de vista, quienes no crecen serán colonizados por colonizadores de países con escasos recursos económicos:

Por otro lado, las "naciones pobres, con individuos desnutridos y con abundantes muestras de carencia de bienes materiales, de higiene, inestabilidad política y social" (ídem), pero en los que la población si crece son sociedades en expansión, sanas que irán colonizando a las que se van extinguiendo. Sin embargo, a muchos de estos países se les ha hecho creer que están "sobrepoblados" y se les han aplicado políticas de reducción de la población, poniéndolas en el camino de la extinción... (CECOR, 2009: 78, v. 5).

Desde esta perspectiva, los hijos(as) son importantes, en tanto ayudan a no ser invadidos por estos "individuos desnutridos" y llenos de carencias. Los pueblos que no se reproduzcan, de acuerdo a esta argumentación, están obligados a desaparecer por la expansión de los(as) inmigrantes. Para este autor, los países con mayor desarrollo económico, aunque tengan una población "bien alimentados, bien vestidos y con claras manifestaciones de poseer abundantes bienes materiales" (CECOR, 2009: 78 , v. 5), tienen la particularidad de no crecer (ídem). El problema de esto es que, aunque se tengan bienes materiales, no tendrán niños y jóvenes propios que sustituyen a los mayores que van muriendo (ídem).

Este argumento queda "como anillo al dedo" para Costa Rica, país que, como ya se sabe, tiene un importante problema no resuelto de xenofobia (Sandoval, 2006) ${ }^{10}$.

Este razonamiento, es pobre en argumentaciones y fácilmente rebatible. Si los(as) niños(as) de los países "pobres" están

10 De acuerdo con Herring y Bonilla (2009: 3) para Costa Rica, los inmigrantes nicaragüenses representaron en el Censo del 2000, cerca de 226 mil, es decir, "el $6 \%$ de la población total o el $77 \%$ de los extranjeros de Costa Rica" (ídem). Como ellos mismos afirman esta población probablemente esté subestimada en esos cálculos, pues se habla de que podrían ser de 400 mil personas. 
desnutridos(as), con pocas posibilidades de educación, reproduciendo el círculo de la pobre$\mathrm{za}$, esto carece de total atención por quienes escogieron este razonamiento. La posibilidad de darle mejor calidad de vida a todas las personas, tampoco es tema de interés. Si las mujeres que se embarazan, deben posponer su vida profesional al cuidado de muchos(as) niños(as), esto tampoco pareciera importante ${ }^{11}$.

Es poco probable que la explicación de eliminar la anticoncepción de la vida de las personas en Costa Rica (aun en la población católica) para evitar que otros(as) nos colonicen, tenga fuerza argumentativa, pues como se dijo anteriormente, la prevalencia anticonceptiva en el país es de $80 \%$ (Chen, op. cit.). Pero en un país tan xenofóbico como el nuestro, el recurso del miedo a la otredad y la exacerbación de las angustias modernas pueden calar en las subjetividades de ciertas personas de forma penetrante. Es más probable que el argumento se utilice como gancho emocional que invoque hacia la hostilidad y/o repugnancia hacia los(as) extranjeros(as), más que hacia un argumento realmente fuerte para oponerse a la anticoncepción ${ }^{12}$.

\section{CONCLUSIONES}

Los textos analizados presentan argumentos contradictorios, como fue señalado anteriormente, pues con alguna frecuencia se cae en incoherencias, no menores, que los vuelven confusos. Por ejemplo, aunque en los textos la sexualidad es vista como ahistórica y natural,

11 Se ha visto que para que las mujeres tengan más oportunidades y no sigan reproduciendo niveles de pobreza, ellas deben reducir el número de hijos y posponer la edad en la que tienen su primer embarazo (Lamas, 2007: 137; Arriagada, 2007: 15).

Como dice Sandoval (Op. cit., 14): "Los excluidos suelen ser considerados sospechosos de quebrar normas y tabúes. Se activan entonces, complejas relaciones entre orden social, estética y moralidad, de tal forma que los grupos excluidos no sólo son percibidos como violadores de las leyes y las normas, sino como particularmente no "limpios". Sin embargo, tales procesos de exclusión no producen vergüenza o culpa, pues la estigmatización es deificada y naturalizada a través de la "etnicización" o cosificación de los "otros"”. también se le reconoce a la misma un espacio de influencia social. Desde el inicio hasta el final, la diversidad o las diferencias son vistas no solo como perniciosas y es propuesta como la causante de crisis.

La visión de clandestinidad, también imprime una marca a los documentos, al plantear que ciertos temas solo deben ser tratados en situaciones muy específicas. Por lo tanto, la sexualidad sigue teniendo, para los textos, un aura enigmática, no solo por lo vago que está tratado el tema teóricamente, sino porque se dice que esta es un "misterio sagrado"; ella es presentada en el documento como un acertijo a resolver o como un rompecabezas del que se espera tener todas las piezas. La brisa de un secretismo confuso, permea su lectura.

Todas las formas de sexualidad no heterosexual son propuestas como negativas. Con la trillada frase "odiar el pecado, pero amar el pecador" (CECOR, 2009: 24, v. 6), se cree que se soluciona todo en materia de diversidad sexual. Por si fuera poco, se recomienda no hablar del tema y cuando se menciona se recomienda la terapia.

El "truco de Dios", término utilizado por Donna Haraway para describir "la producción de conocimiento desde arriba, desde un lugar que niega la situación del sabio individual en un mundo real y problemático" (Fausto-Sterling, 2006: 21), resulta muy útil en estos textos y en otros de carácter religioso, que procuran que las personas actúen de cierta manera, para respetar "la voluntad divina". Este término, puede contener tan variadas "voluntades" que su uso resulta ambiguo y cambiante de acuerdo a quien lo utilice. Aun así, su aplicación continúa hoy siendo inmensamente efectiva.

Parece importante recordar que al hablar de sexualidad no se puede desconocer la construcción cultural y social que esta ha experimentado $y$ la variabilidad de vivencias $y$ prácticas que conviven en las sociedades, no solo por el periodo histórico vivido, sino también por otras especificidades que se interrelacionan como lo son el género, la raza, la clase social, las creencias religiosas o mágicas, entre muchas otras. Como dice Weeks (1998), la sexualidad es una "unidad ficticia que alguna 
vez no existió y que en algún momento en el futuro tal vez de nuevo no existirá".

Promover reflexiones críticas, históricas $y$ con perspectiva de género, son fundamentales para cuestionar dogmatismos religiosos e ideológicos que no están interesados en promover cambios, que parecen estar ciegos a las realidades de las mujeres y los hombres y que lo único que logran es perpetuar las relaciones existentes de dominación y control sexuales.

El problema aquí no es realizar módulos para trabajar el tema de la sexualidad desde diferentes puntos de vista (por variados que estos sean), el punto cuestionable es más bien, querer negar la realidad sexual de las personas y crear modelos ahistóricos, idílicos, "castos" de los seres humanos, dándole la espalda a las realidades de quienes habitan en el mundo. Por lo tanto, una pregunta necesaria es pensar si las personas a las que van dirigidas los módulos realmente existen o son ilusiones proyectadas por deseos de un grupo religioso. Seguiría siendo contraproducente, perder de vista las realidades sexuales de las personas en Costa Rica, por diversas que parezcan.

Existe para la ciudadanía un reto importante, en el que habría que planear el cómo enfrentarse a estos fundamentalismos moralistas que convergen, entre muchas otros, en nuestras sociedades occidentales. Para nadie es un secreto la fuerte influencia de la jerarquía católica en Costa Rica, país que sigue teniendo una religión en la Constitución Política. Aun así, recientemente, en un artículo de periódico el titular señalaba que el "MEP se desmarca de obispos católicos en educación sexual" (Oviedo, 2010); allí se mencionaba que aunque la presidenta Laura Chinchilla había tenido acercamientos con los obispos católicos, el Ministerio de Educación Pública, se preparará sobre aspectos relacionados con la sexualidad donde "no solo informe sobre la existencia de métodos anticonceptivos, sino que eduque tanto de salud reproductiva como de vínculos afectivos, madurez emocional y placer" (ídem). El plan se empezará a realizar en julio de 2011 $y$ tiene como base los documentos elaborados por el Instituto de Estudios Interdisciplinarios de la Niñez y la Adolescencia de la Universidad
Nacional. Habrá que seguir atentos(as) al desenvolvimiento de estos acontecimientos para procurar mejores vivencias de las sexualidades, sin voltear la mirada hacia lo que quisiéramos que esta fuera.

\section{BIBLIOGRAFÍA}

Arangurem, Jose Luis. "La religión, hoy". Formas modernas de religión. Rafael Díaz-Salazar, Salvador Giner, Fernando Velasco (eds.). Madrid, España. Alianza Universal, 1996: 21-37.

Arriagada, Irma. "Familias Latinoamericanas: cambiantes, diversas y desiguales". Papeles de Población 053. Toluca, México. Universidad Autónoma del Estado de México, julio-setiembre 2007: 9-22.

Ávalos, Ángela. "Guías sexuales de Iglesia prohíben condón y píldora”. Periódico La Nación. Sección El País. Costa Rica, 2009a. [En línea]. En: <http:// wvw.nacion.com/ln_ee/2009/mayo/15/ pais1965243.html> [Revisado el 20 de diciembre de 2010].

Ávalos, Ángela. "Colegios católicos usarán guías sexuales aprobadas por obispos”. La Nación. Sección El País. Costa Rica, 2009b. [En línea]. En: <http:// www.nacion.com/ln_ee/2009/mayo/25/ pais1975485.html> [revisado el 20 de diciembre de 2010].

Butler, Judith. El género en disputa: el feminismo y la subversión de la identidad. España: Paidós, 2007.

Chen Mok, Mario et ál. Salud reproductiva y migración nicaragüense en Costa Rica, 1999-2000: resultados en una encuesta nacional de salud reproductiva. San José: Copieco de San Pedro, 2001.

Conferencia Episcopal de Costa Rica. "Cuidados, afecto y amor: 0 a 6 años de edad". Serie Amor y Sexualidad 1. San José, Costa Rica: Editorial CECOR, Conferencia Episcopal de Costa Rica, 2009.

Conferencia Episcopal de Costa Rica. "La edad de la Inocencia: 7 a 9 años de edad". Serie Amor y Sexualidad 2. San José, 
Costa Rica: Editorial CECOR, Conferencia Episcopal de Costa Rica, 2009.

Conferencia Episcopal de Costa Rica. "Descubriéndonos compañeros y amigos: 10 a 12 años de edad". Serie Amor y Sexualidad 3. San José, Costa Rica: Editorial CECOR, Conferencia Episcopal de Costa Rica, 2009.

Conferencia Episcopal de Costa Rica. "Compañeros y amigos: 13 a 15 años de edad". Serie Amor y Sexualidad 4. San José, Costa Rica: Editorial CECOR, Conferencia Episcopal de Costa Rica, 2009 .

Conferencia Episcopal de Costa Rica. "Compañeros, amigos y novios: 16 a 17 años de edad". Serie Amor y Sexualidad 5. San José, Costa Rica: Editorial CECOR, Conferencia Episcopal de Costa Rica, 2009.

Conferencia Episcopal de Costa Rica. "Colaborando con la vida: 18 años y más de edad". Serie Amor y Sexualidad 6. San José, Costa Rica: Editorial CECOR, Conferencia Episcopal de Costa Rica, 2009.

Fausto-Sterling, Anne. Cuerpos sexuados: la política de género y la construcción de la sexualidad. España: Editorial Melusina, 2006.

Foucault, Michelle. Historia de la sexualidad I. México: Siglo XXI, 1986.

Gamboa, Isabel. El sexo como Lo cura. En el Hospital Psiquiátrico. San José, Costa Rica: Grafos Litografía, 2009.

Giddens, Anthony. La transformación de la intimidad: sexualidad, amor y erotismo en las sociedades modernas. Tercera edición. Madrid, España: Ediciones Cátedra, 2000.

Jiménez Guzmán, María Lucero y Tena Guerrero, Olivia. "Notas sobre negociación coital”. Elementos para el análisis ético de la reproducción. México: Programa Universitario de Estudios de Género, Universidad Nacional Autónoma de México. 2001.

Jiménez Roger, Beatriz y Osuna López, María Carmen. "Religión y cambio cultural: el caso de los jóvenes". El fenómeno religioso: Presencia de la religión y de la religiosidad en las sociedades avanzadas. II Jornadas de Sociología. 13 y 14 de junio 2007. [En línea]. En: <http://www. centrodeestudiosandaluces.info/cursos/ adjuntos/4483179.pdf> [Revisado el 21 de mayo de 2010].

Herring, Andrew A. y Bonilla, Roger E. "Inmigrantes nicaragüenses en Costa Rica: estado y utilización de servicios de salud". Revista Población y Salud en Mesoamérica 7 (1). Costa Rica: Universidad de Costa Rica, juliodiciembre 2009: 1-19.

Lamas, Marta. "Género, desarrollo y feminismo en América Latina". Pensamiento Iberoamericano 0. Agencia Española de Cooperación Internacional, 2007: 133-152.

Maffia, Diana y Cabral, Mauro. "Los sexos ¿son o se hacen?”. Sexualidades migrantes: género y transgénero. Diana Maffia (comp.). Buenos Aires, Argentina. Editorial Feminaria, 2003: 86-96.

Organización Panamericana de la Salud. "La salud en las Américas". Publicación Científica y Técnica 587. Organización Panamericana de la Salud, 2002.

Oviedo, Esteban. "MEP se desmarca de obispos católicos en educación sexual". La Nación. Sección El País. Lunes 13 de diciembre, 2010: 4A.

Rubin, Gayle. "Reflexionando sobre el sexo: notas para una teoría radical de la sexualidad". Placer y peligro: explorando la sexualidad femenina. Carol Vance (comp.). Madrid, España: Editorial Revolución SA, 1989: 113-190.

Sandoval García, Carlos. Otros amenazantes: los nicaragüenses y la formación de identidades nacionales en Costa Rica. San José, Costa Rica: Editorial de la Universidad de Costa Rica, 2006.

Vendrell Ferré, Joan. "La centralidad de la sexualidad en la era moderna". Sexualidades diversas: aproximaciones para su análisis. Gloria Careaga 
y Salvador Cruz (coords.). México: Programa Universitario de Estudios de Género, Universidad Nacional Autónoma de Género. 2004.

Wikipedia. Intersexualidad. [En línea]. En: <http://es.wikipedia.org/wiki/ Intersexualidad $>$ [Revisado el 20 de diciembre de 2010].
Weeks, Jeffrey. Sexualidad. México: PaidósPrograma Universitario de Estudios de Género, Universidad Nacional Autónoma de México. 1998.

Fecha de ingreso: 27/01/2011

Fecha de aprobación: 26/05/2011 
